\title{
Development of Parallel Algorithms for Calculation of Backscattering Characteristics of Complex Shape Objects
}

\author{
V. B. Suchkov, M. I. Baev, A. K. Likhoedenko \\ Department of Special machinery, Bauman Moscow State Technical University, Moscow, 105005, \\ Russia
}

Keywords: scattering field, physical optics, radar cross section, Open Multi-Processing (OpenMP) library, parallel algorithms.

Abstract. A programming technique, which is carried out using the Open Multi-Processing (OpenMP) library and can be employed to assess scattering fields from electrically large complex shape objects, is developed and presented in this paper. The basic idea is to employ the OpenMP library to accelerate paths tracing for physical optics (PO) and shooting and bouncing rays (SBR) methods. The procedure of OpenMP-based paths tracing is divided into two steps, which are shadow faces removal and reflected rays searching. In addition, OpenMP speedup scheme has been proposed to improve the efficiency of PO algorithm.

\section{Introduction}

The ability to process large amounts of information is the main attraction of modern radar technologies:

- radar images processing of electrically large targets with high resolution;

- SAR (Synthetic Aperture Radar) simulation;

- automatic target recognition;

- calculation of radar cross section (RCS) of complex shape objects with electrically large size.

SAR images of a tank and an airplane for a radar wave center frequency of $10 \mathrm{GHz}$ and a resolution of $10 \mathrm{~cm}$ given by Chirp Scaling Algorithm (CSA) [1] are shown in figure $1(\mathrm{a}, \mathrm{b})$. RCS diagram of a missile with a length of $4.2 \mathrm{~m}$ for a radar frequency of $10 \mathrm{GHz}$ calculated by PO method with use of SBR technology is shown in figure 2.

Scattering fields target simulation needs to describe various mechanisms of electromagnetic scattering waves, such as edge diffraction, multiple scattering, etc. Modern electromagnetic computing methods for SAR simulation and RCS prediction can be sped up using parallel multiprocessing algorithms, which are mainly suitable for the high frequency calculation.

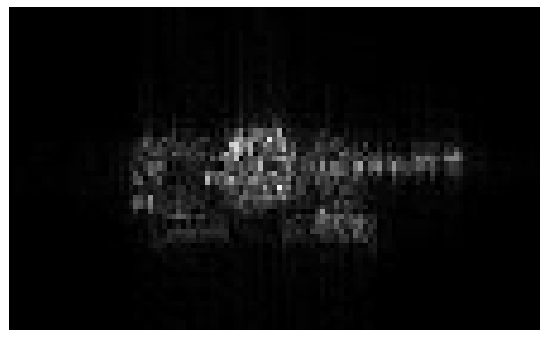

(a)

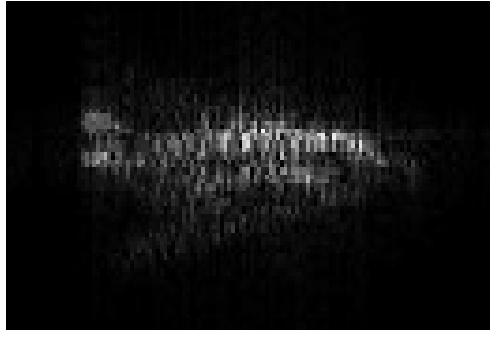

(b)

Fig.1: Radar images of electrically large targets with high resolution (a - radar image of tank, b- radar image of airplane) 


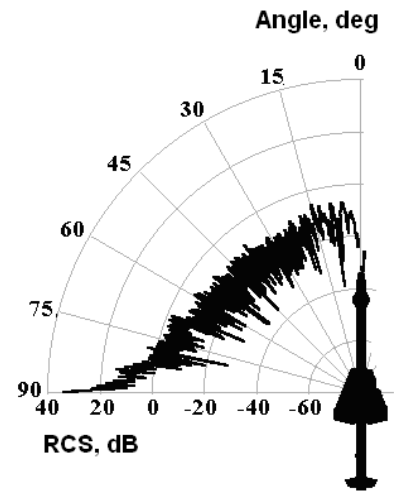

Fig.2: RCS diagram of electrically large missile

In view of the research situation presented above, this paper proposes a method of image simulation and RCS prediction based on parallel multiprocessing algorithms for realization of ray tracings of PO and SBR methods.

\section{Discussed Problems}

Modern exact methods such as the method of moments (MoM) and Multilevel Fast Multipole metgod (MLFMM) [2] are suitable for a very accurate calculation of RCS and high resolution radr images of complex shape targets. However, even with the use of MLFMM, the computation and memory efforts are immense at higher frequencies [3]. So asymptotic methods based on physical optics (PO) and physical theory of diffraction (PTD) are often applied for the modeling of electrically large objects. And using of asymptotic methods based on polygonal models of targets. It is supposed that all elements of a radar target are set on the basis of polygonal models which can be generated in various CAD. Thus each constructive element of a radar target is represented as set of the elementary reflectors allocated a set of electrophysical and statistical parameters, describing its unique properties. Polygonal models are formed in the environment of modern CAD systems like Creo or SolidWorks. CAD systems describe an external surface of object as triangular meshes, i.e. as base spatial elements of polygonal model the plane triangular plates definitely joined with each other are used. As an example on fig. 3 polygonal models of a missile with different number of triangles are shown.

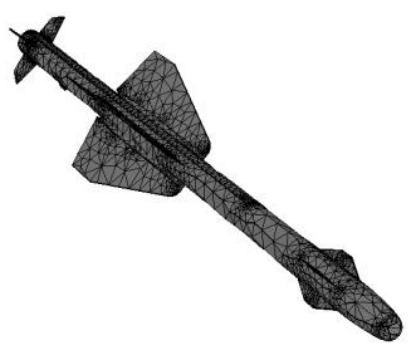

(a)

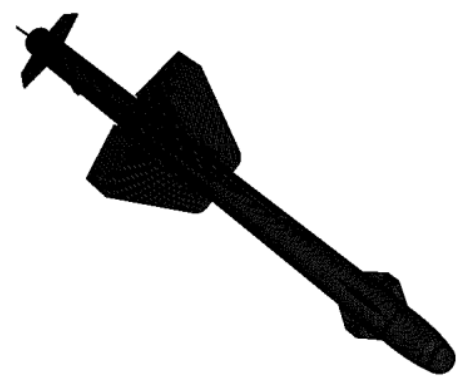

(b)

Fig. 3. Polygonal models of a missile generated Creo

( $a$ - number of triangles is $12000, b$ - number of triangles is 143000)

According to asymptotic methods (PO, PTD and SBR) electromagnetic scattering field of the object set by the polygonal model, the resulting field of the scattering received in the aperture of reception antenna of radar, is represented as superposition three components [4,5]:

$$
\dot{E}_{s c \Sigma}=\dot{E}_{t r . \Sigma}+\dot{E}_{e d . \Sigma}+\dot{E}_{r r f . \Sigma}
$$

where $\dot{E}_{s c \Sigma}$ - the resulting field of scattering of the object received in the aperture of reception antenna, $\dot{E}_{t r . \Sigma}$ - resulting field of scattering of all plane triangular elements forming polygonal 
model (PO-method), $\dot{E}_{e d . \Sigma}$ - resulting field of scattering of sharp edges of polygonal model (PTDmethod), $\dot{E}_{r r f . \Sigma}$ - superposition of fields of scattering of triangles for which sources of excitation of secondary waves are induced due to double reflections from other triangles of polygonal mode (ray tracings between triangles, SBR-method).

At formation of mathematical model of a field of scattering has no difference of an element of polygonal model (a triangle or an edge). Therefore it is possible to present size of intensity of a resulting field of scattering of object (1) as superposition of fields of scattering of all elements (elementary reflectors) to polygonal model of object:

$$
E_{p \Sigma}=\sum_{m=0}^{N_{\Sigma}-1} E_{s m},
$$

where m - index of elementary reflector (ER), $\dot{E}_{s m}=\vec{E}_{s m} \vec{E}_{0 m}^{s}$ size of intensity of a resulting field of m-th ER received in the aperture of receiving antenna, $N_{\Sigma}=N_{t r}+N_{e d}+\tilde{N}_{t r(r r f)}$ - total number of the reflectors participating in scattering of electromagnetic waves.

To calculate resulting electromagnetic scattering field of the object (2) by methods PO, PTD and SBR an implementation of two steps are required:

- shadow triangles removal, because PO current density is null in the triangles not illuminated by the source and shadowed by other triangles;

- reflected rays searching (for finding the reflection rays to use SBR method illuminated triangles should be tested one by one).

So for high frequencies of radar a size of object becomes electrically large and number of triangles of polygonal model is extremely rising (fig. 3(b)). So it is necessary to improve algorithms of shadow triangles removal and reflected rays searching with the aim to speed up calculations of large arrays of triangles.

\section{Parallel Multiprocessing Algorithm of Shadow Triangles Removal}

Sources of fields of scattering of the electromagnetic waves, distributed on a surface of object of the complex form in approach of PO and PTD methods, make sense only in those areas on surface of object $S$ which are shined on the part of the transmitter of a radar and are not shaded on the part of the receiver of a radar. Step function of shading from radar of triangle $T_{j}(j-$ an index of a triangle in a file of triangular elements of polygonal model), belonging to surface $S$, is represented in the following view:

$$
\begin{aligned}
& \delta_{t r}\left(T_{j}\right)=1, T_{j} \in L_{i}^{+}, \\
& \delta_{t r}\left(T_{j}\right)=0, T_{j} \notin L_{i}^{+}
\end{aligned}
$$

where $L_{i}^{+}$- area on surface $S$, shined on the part of a radar.

The analysis of conditions of shadings and maskings of elements of polygonal model is carried out in a cycle on all triangular elements. The triangular element is considered visible if it satisfies to a condition of not masking itself, and also to a condition of not shading by other elements. For a triangular element visible by radar function of shadings and maskings (3) is accepted equal $\delta_{t r}=1$. The algorithm of search of not masking triangles of model represents a cycle on all triangular sides $T_{j}$ of digital model of object of a location. On each iteration of a cycle the condition of not masking a triangle $T_{j}$ is checked and if the condition is carried out the triangle is accepted as not-masking. Otherwise, the triangle $T_{j}$ is considered self-maskable and is missed. The condition of not masking a triangle concerning radar is defined as $\vec{r} \vec{n}<0$, where $\vec{n}$ - a vector of a normal to a plane of a triangle, $\vec{r}$ - the vector which has been directed from the radar in a middle point of triangle. In other words, a triangle is not masking if the corner between vectors $\vec{n}$ also $\vec{r}$ is not acute. 
The algorithm of search not shaded elements represents a cycle on all not masking triangles $T_{j}$ of digital model of object of a location. On each iteration of a cycle for a triangle $T_{j}$ first other triangle shading it is searched. This search is realized as an internal cycle on all not masking triangles $T_{k}$, except for $T_{j}$. If the shading triangle is not found, the triangle $T_{j}$ is considered not shaded and its function of shading $\delta_{t r}\left(T_{j}\right)=1$, otherwise the triangle $T_{j}$ is considered shaded and is missed.

Simulation on the given algorithm is the most labour-consuming problem, since in it there is an internal cycle, and to confirm that the triangle $T_{j}$ is not shaded, it is necessary to pass on all not masking triangles $T_{k}$, except for $T_{j}$, therefore the increasing of quantity of triangles leads to increasing of time of process of calculations. With increase in total quantity of not masking triangles time of similation grows even more strongly. The algorithm of check on shading of a triangle $T_{1}$ by other triangle $T_{2}$ looks as follows (fig. 4). At the first step the vector $\vec{r}_{1}=M_{1}-P$ from the phase center of antenna of radar in an middle point of first triangle $\mathrm{M}_{1}$ is directed. At the second step the distance between the phase center of the antenna and a triangle $T_{1} R_{1}=\left|M_{1}-P\right|$ is calculated. At the third step calculation of a point of crossing $P_{r}$ of a vector $\vec{r}_{1}$ with a plane of the second triangle $T_{2}$ is carried out:

$$
P_{r}=P-\frac{\left(\vec{n}_{2} \cdot \vec{r}_{2}\right)}{\left(\vec{n}_{2} \cdot \vec{r}_{1}\right)} \vec{r}_{1},
$$

where $\vec{n}_{2}$ - vector of a normal to a plane of the second triangle $\mathrm{T}_{2}, \vec{r}_{2}=M_{2}-P$ - the vector which has been directed from the phase center of the antenna in an middle point of the second triangle

At the fourth step check of a belonging of a point of crossing $P_{r}$ to the second triangle $T_{2}$ is carried out (fig. 5). The condition $P_{r} \in T_{2}$ (belongings of a point $P$ to a triangle $P_{1} P_{2} P_{3}$ ) refers to as a condition of incidence. The given condition consists that the point $P_{r}$ belongs to a triangle $P_{1} P_{2} P_{3}$ if the next condition is carried out (fig. 9):

$$
\left\{\begin{array}{l}
\left(\vec{n}_{2}\left[\vec{p}_{1} \times \vec{p}_{2}\right]\right) \geq 0, \\
\left(\vec{n}_{2}\left[\vec{p}_{2} \times \vec{p}_{3}\right]\right) \geq 0, \\
\left(\vec{n}_{2}\left[\vec{p}_{3} \times \vec{p}_{1}\right]\right) \geq 0,
\end{array}\right.
$$

where $\vec{p}_{1}=P_{1}-P_{r}, \vec{p}_{2}=P_{2}-P_{r}, \vec{p}_{3}=P_{3}-P_{r}$.

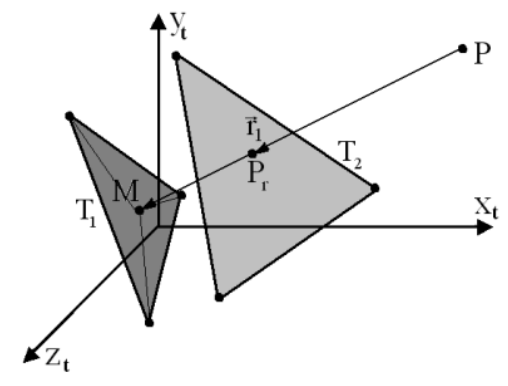

Fig. 4 The scheme of shading of triangle $T_{1}$ by triangle $T_{2}$ 


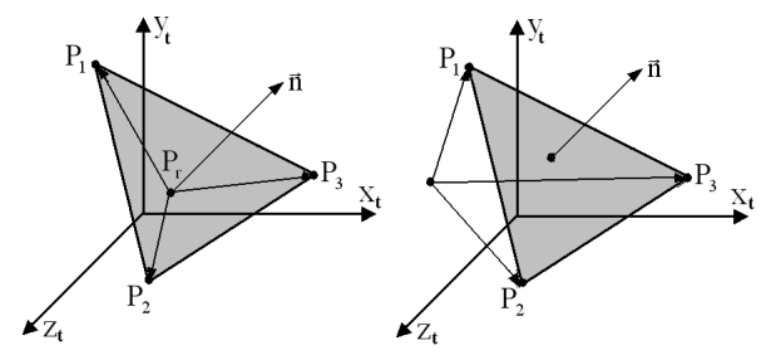

Fig. 5. Condition of a belonging of a point $\mathrm{P}$ to triangle (at the left - the point belongs to a triangle, on the right - a point outside a triangle)

It is obvious that the reference in zero, for example, the first inequality will mean, that the point $P$ lays on a piece $P_{1} P_{2}$. If the point $P_{r}$ belongs to a triangle $T_{2}$ at the fifth step the distance between the phase center of the antenna and a triangle $T_{2}$ is calculated: $R_{2}=\left|P_{r}-P\right|$. In the conclusion at the sixth step direct check of a triangle $T_{2}$ on shading is carried out, by comparison of distances $R_{1}$ and $R_{2}$. It is considered that the triangle $T_{2}$ shades a triangle $T_{1}$, if $R_{2}>R_{1}$.

To speed up calculations of shadow triangles removal we used the Open Multi-Processing (OpenMP) library which describes set of directives of the compiler, library procedures and variables of an environment which are intended for programming of multiline applications on the multiprocessor systems with the general memory. The OpenMP technology is expansion of the C/C language ++ , supplementing it with special directives of a preprocessor (pragmama) which allow to use computing abilities of the multi-core central processor. The announcement of the directive happens as follows \#pragma omp parallel. Then there is a code placed in braces " $\{$ " and " $\}$ " which carries out in parallel on all cores which are available on CPU. The parallelized code of one of the most labor-consuming functions of the program connected with search of shadow triangles removal is given in fig. 6 .

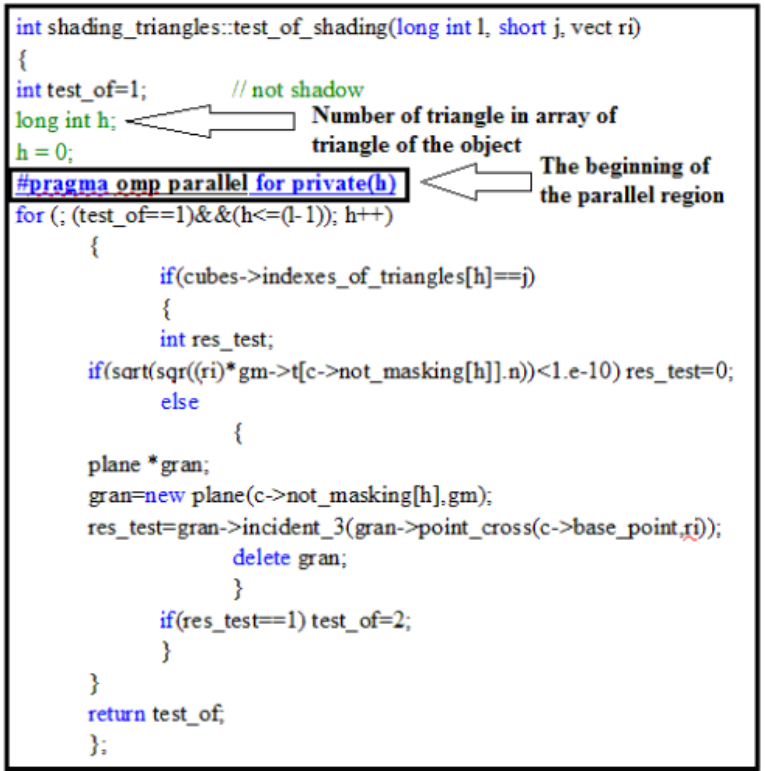

Fig. 6. Programming code of function of shadow triangles removal with use of OpenMP library

The 'test_of_shading' function (fig. 6) checks of a not-shading triangles. The Open MP technology possesses the special directive \#pragma omp parallel for which distributes performance of streams. At the end of a cycle there is a synchronization of all streams, it occurs implicitly, but there is a directive 'nowait' capable to cancel synchronization. Time of implementation of the program for calculation of shadow triangles removal of missile (fig. 3b) with size 4,2 $\mathrm{m}$ (for large 
number of triangles) is assessed for using of OpenMP library and without it (table 1). All calculations are provided on IBM PC with multicore processor Intel Core i7 and 3 GGb of RAM.

Table 1 - Time of calculation of not-shading triangles of a missile polygonal model for different types of algorithm of shadow triangles removal

\begin{tabular}{|c|c|}
\hline Without OpenMP library, min. & $\begin{array}{c}\text { With use of Open MP } \\
\text { library, min. }\end{array}$ \\
\hline 41 & 22 \\
\hline
\end{tabular}

Thus, it is visible that the parallel version is carried out almost twice faster (if to be more exact at 1,86 times).

\section{Conclusions}

It is shown that the most resource-intensive part of the program of calculation of RCS target on the base of Po and CBR methods is algorithm of definition of not shaded triangles For increase of speed of calculations of shadow triangles removal the algorithm of parallel calculations with use of the OpenMP library is offered. As a result it is shown that using of OpenMP library provides decreasing of time of calculations at least two times by comparing common algorithm.

\section{Acknowledgements}

This study was performed under project no. 1776, order no. 8.1776.2014/K for research in the project part of the State research work of the Ministry of Education and Science of the Russian Federation.

\section{References}

[1] I. G. Cumming, F. H. Wong Digital Processing of Synthetic Aperture Radar Data: Algorithms and Implementation. Boston; London: Artech House, 2005. - 625 p.

[2] Gibbson W.C. The Method of Moments in Electromagnetics. Boca Raton: Chapman\&Hall/CRC, 2008.- 594 p.

[3] Tian-Qi Fan \& Li-Xin Guo. OpenGL-Based Hybrid GO/PO Computation for RCS of Electrically Large Complex Objects. IEEE Antennas and wireless propagation letters, Vol. 13, pp. 666-669, 2014.

[4] Shyh-Kang Jeng. Near-field scattering by physical theory of diffraction and shooting and bouncing rays. IEEE Trans. on Antennas and Propagation, Vol. 46, № 4, pp. 551-558, 1998.

[5] Ling H., Chou R.C.,Lee S.W Shooting and bouncing rays: Calculating the RCS of an arbitrarily shaped cavity. IEEE Trans. Antennas and Propagation, Vol. 37,. pp. 194-205, 1989. 